PROCEEDINGS OF THE

AMERICAN MATHEMATICAL SOCIETY

Volume 126, Number 1, January 1998, Pages 289-296

S $0002-9939(98) 03978-1$

\title{
DEHN FILLING, REDUCIBLE 3-MANIFOLDS, AND KLEIN BOTTLES
}

\author{
SEUNGSANG OH
}

(Communicated by Ronald Fintushel)

\begin{abstract}
Let $M$ be a compact, connected, orientable, irreducible 3-manifold whose boundary is a torus. We announce that if two Dehn fillings create reducible manifold and manifold containing Klein bottle, then the maximal distance is three.
\end{abstract}

\section{INTRODUCTION}

Let $M$ be a compact, connected, orientable, irreducible 3-manifold such that $\partial M$ is a torus. The slope of an essential simple loop on $\partial M$ is its isotopy class, and if $\pi$ and $\gamma$ are two slopes on $\partial M$ then $\triangle=\triangle(\pi, \gamma)$ will denote their minimal geometric intersection number. Let $M(\pi)$ denote the manifold obtained from $M$ by $\pi$-Dehn filling, that is, by attaching a solid torus $V_{\pi}$ to $M$ along $\partial M$ so that the boundary of a meridian disk is identified with $\pi$, and similarly for $\gamma$.

There are many results on $\triangle(\pi, \gamma)$ for two distinct slopes $\pi$ and $\gamma$ on $\partial M$, for example [BZ1], [CGLS], [Go1], [GLu1], and [Wu1]. Especially Gordon and Luecke [GLu2] have shown that if both $M(\pi)$ and $M(\gamma)$ are reducible, then $\triangle \leq 1$, and $\mathrm{Wu}[\mathrm{Wu} 2]$ and $\mathrm{Oh}[\mathrm{Oh}]$ proved independently that if $M$ is hyperbolic and $M(\pi)$ is reducible while $M(\gamma)$ contains an incompressible torus, then $\triangle \leq 3$. In this paper we consider the situation where $M(\gamma)$ contains an embedded Klein bottle.

Theorem 1.1. Let $M$ be a hyperbolic 3-manifold. If $M(\pi)$ is reducible and $M(\gamma)$ contains a Klein bottle, then $\triangle(\pi, \gamma) \leq 3$.

It is still unknown whether or not the bound 3 is best possible. This result gives us a partial improvement to Theorem 0.1(2) of [BZ2], dealing with reducible and finite Dehn fillings.

Corollary 1.2. If $M$ is hyperbolic, $M(\pi)$ is reducible and $M(\gamma)$ is a Seifert fiber space over the 2-sphere with three exceptional fibers of orders $2,2, n$, then $\triangle(\pi, \gamma) \leq$ 3.

We will be following closely the argument in [Oh] and will hereafter assume familiarity with this paper. To obtain a contradiction, we shall suppose that $\triangle(\pi, \gamma) \geq 4$. Let $\widehat{Q}$ be a reducing sphere in $M(\pi)$ which intersects $V_{\pi}$ in a family of meridian

Received by the editors April 8, 1996 and, in revised form, May 31, 1996.

1991 Mathematics Subject Classification. Primary 57M25, 57M99, 57N10.

Key words and phrases. Dehn filling, reducible, Klein bottle, 3-manifold.

(C)1998 American Mathematical Society 
disks. We choose $\widehat{Q}$ so that $Q=\widehat{Q} \cap M$ has the minimal number, say $q$, of boundary components. Similarly we choose $\widehat{S}$, a Klein bottle in $M(\gamma)$ in such a manner.

By an isotopy of $Q$, we may assume that $Q$ and $S$ intersect transversely, and $Q \cap S$ has the minimal number of components. Then no circle component of $Q \cap S$ bounds a disk in $Q$ or $S$, and no arc of $Q \cap S$ is boundary parallel in $Q$ or $S$. The boundary of a regular neighborhood of $\widehat{S}$ is a torus $\widehat{T}$, meeting $V_{\gamma}$ in $t=2\left|\widehat{S} \cap V_{\gamma}\right|$ points in $M(\gamma)$. Note that $\widehat{T}$ is not necessarily incompressible. Now we obtain a graph $G_{Q}$ in $\widehat{Q}$ by taking $\widehat{Q} \cap V_{\pi}$ as its fat vertices and the $\operatorname{arcs}$ in $Q \cap T$ as its edges. Similarly we obtain the graph $G_{T}$ in $\widehat{T}$. Note that each fat vertex of $G_{Q}$ $\left(G_{T}\right)$ intersects each fat vertex of $G_{T}$ (respectively $G_{Q}$ ) exactly $\triangle$ times. Number the components of $\partial Q 1,2, \ldots, q$ successively along $\partial M$, and similarly number the components of $\partial T 1,2, \ldots, t$. In this way each end of each edge of $G_{Q}\left(G_{T}\right)$ has a label, namely the number of the corresponding fat vertex of $G_{T}$ (respectively $G_{Q}$ ). When traveling around a fat vertex of $G_{Q}$, the labels appear as $1, \ldots, t$ repeated $\triangle$ times and similarly for a fat vertex of $G_{T}$. Assigning orientations to $\widehat{Q}$ and $\widehat{T}$ allows us to refer to + and - vertices of $G_{Q}\left(G_{T}\right)$, according to the sign of the corresponding intersection with the core of $V_{\pi}$ (respectively $V_{\gamma}$ ). If two vertices have the same sign they are called parallel, otherwise antiparallel. The orientability of $Q, T$ and $M$ give us the following parity rule : an edge connects parallel vertices on one graph if and only if it connects antiparallel vertices on the other. As is done above, define the labelled graphs $G_{Q}^{S}$ in $\widehat{Q}$ and $G_{S}$ in $\widehat{S}$ coming from the intersection of $Q$ and $S$.

The author is indebted to his advisor Cameron Gordon for many stimulating conversations.

\section{Preliminaries}

In this section we define the concept of a Scharlemann cycle and a $(k) x$-web, and introduce some combinatorial techniques developed in other articles.

Let $G$ be the graph $G_{Q}$ or $G_{T}$, and $x$ a label of $G$. An $x$-edge in $G$ is an edge with label $x$ at one endpoint. An $x$-cycle is a cycle of $x$-edges of $G$ such that all the vertices are parallel and all the edges can be oriented so that the tail of each edge has label $x$. A Scharlemann cycle is an $x$-cycle that bounds a disk face of $G$. A Scharlemann cycle with exactly two edges is called an $S$-cycle. Note that by construction, $G$ has no face with only one edge.

A $(k) x$-web is a connected subgraph $\Sigma$ of $G_{Q}$ such that all the edges of $\Sigma$ are $x$-edges, all the vertices of $\Sigma$ are parallel, and all but possibly $k$ edges with label $x$ at the vertices of $\Sigma$ connect them to the vertices of $\Sigma$. Such exceptional edges are called ghost edges, and their endpoints in $\Sigma$ are called ghost vertices. A great (k) $x$-web satisfies the additional condition that there is a component $U$ of $\widehat{Q}-\Sigma$ such that all the vertices of $G$ in $\widehat{Q}-U$ have the same sign.

We hereafter assume that $q \geq 3$ because of Lemma 2.3 of [BZ3]. We have the following lemma from the argument of Proposition 1.3 of [GLi].

Lemma 2.1. $G_{T}$ (hence $G_{S}$ ) cannot have $q$ mutually parallel edges.

If we assume that $t \geq 4$, then we have the following two lemmas; Lemma 2.2 is Lemmas 2.6 and 2.7 of $[\mathrm{Oh}]$ and Lemma 2.3 can be obtained from Lemmas 2.1 2.4 of [Wu1]. 
Lemma 2.2. (1) $G_{Q}$ has at most two $S$-cycles on disjoint label pairs.

(2) $G_{Q}$ has at most $t / 2+2$ mutually parallel edges connecting parallel vertices. Furthermore, if $t \equiv 2(\bmod 4)$, then $G_{Q}$ cannot have $t / 2+2$ mutually parallel edges connecting parallel vertices.

Lemma 2.3. (1) $G_{T}$ cannot contain two $S$-cycles on distinct label pairs.

(2) $G_{T}$ has at most $q / 2+1$ mutually parallel edges connecting parallel vertices. Furthermore, if there are such $q / 2+1$ edges, then first two or last two of these edges form an $S$-cycle in $G_{T}$.

\section{Proof of Theorem 1.1 (The Case $t \geq 6$ )}

In this section we prove Theorem 1.1 when $t \geq 6$. As the argument in the case $t=2$ or 4 is quite different, we handle it separately in Section 4 .

We assume familiarity with the terminology of [GLu1, Section 2.1] and the more generalized terminology discussed in [GLu2]. Proposition 3.1 of [Oh], which is the analog of Proposition 3.1 of [GLu2], is still true in our case, indeed it works for any $\triangle \geq 2$. Hence we get the following proposition;

Proposition 3.1. Either $G_{Q}$ contains a great $(k) x$-web, or for all $\{1, \ldots, q\}$-types, $\tau$, there are at least $k t$ faces of $G_{T}(L)$ representing $\tau$.

Let $k$ be the smallest number greater than $\triangle / 2$. Now Theorem 1.1 is broken into two cases, which will be carried out in the rest of this section. For a graph $\Gamma$, the reduced graph $\bar{\Gamma}$ of $\Gamma$ is defined to be the graph obtained from $\Gamma$ by amalgamating each family of mutually parallel edges of $\Gamma$ to a single edge. Note that every family of mutually parallel edges of $G_{Q}$ has an even number of edges since $\widehat{T}$ is the boundary of a regular neighborhood of $\widehat{S}$.

Theorem 3.2. $G_{Q}$ cannot contain a great $(k) x$-web.

Proof. Assume for contradiction that there is a great $(k) x$-web $\Sigma$ in $G_{Q}$. We may assume that $\Sigma$ has no separating edge, for if $e$ is an edge of $\Sigma$ such that $\Sigma-e$ has two components then one of them is also a great $(k) x$-web. Let $U$ be a component of $\widehat{Q}-\Sigma$ such that all the vertices of $G_{Q}$ in $\widehat{Q}-U$ have the same sign. Let $\Gamma_{Q}$ be the subgraph $G_{Q}-U$ of $G_{Q}$. Suppose that $U$ is an $n$-gon, i.e. $\bar{\Gamma}_{Q}$ has $n$ boundary vertices. Let $v, e$ and $f$ be the number of vertices, edges and faces of $\bar{\Gamma}_{Q}$ in $\widehat{Q}$. Since each face of $\bar{\Gamma}_{Q}$ is a disk with at least 3 sides, we have $2 e \geq 3(f-1)+n$. Thus

$$
2=\chi(\widehat{Q})=v-e+f \leq v-\frac{e}{3}+1-\frac{n}{3} .
$$

Therefore $2 e \leq 6 v-2 n-6$. We distinguish two cases

(1) Some interior vertex $y$ of $\bar{\Gamma}_{Q}$ has valency at most $\triangle+1$.

There are $\triangle t$ edges in $G_{Q}$ which are incident to $y$ and connect $y$ to parallel vertices. By Lemma $2.2(2), \triangle t \leq(\triangle+1)\left(\frac{t}{2}+2\right)$, i.e. $t<7$ and $t \neq 6$, a contradiction.

(2) All interior vertices of $\bar{\Gamma}_{Q}$ have valency at least $\triangle+2$.

Suppose some boundary vertex $y$ of $\bar{\Gamma}_{Q}$ which is not a ghost vertex has valency at most $\triangle-1$. Then at least $(\triangle-1) t+2$ edges (must be even because $T$ is a double covering of $S$ ) incident to $y$ in $G_{Q}$ connect $y$ to parallel vertices. Again $(\triangle-1) t+2 \leq(\triangle-1)\left(\frac{t}{2}+2\right)$ by Lemma $2.2(2)$. Hence $t<4$. Therefore all boundary vertices of $\bar{\Gamma}_{Q}$ which are not ghost vertices have valency at least $\triangle$. Similarly each boundary vertex of $\bar{\Gamma}_{Q}$ which is a ghost vertex with $i$ ghost edges has valency at 
least $\triangle-i$. Since $\bar{\Gamma}_{Q}$ has at most $k$ ghost edges, we get the following inequality (here $v-n$ is the number of interior vertices):

$$
(\triangle+2)(v-n)+\triangle n-k \leq 2 e .
$$

From two previous inequalities, we finally have $(\triangle-4) v+6 \leq k$, which contradicts that $k \leq \triangle / 2+1$.

It was pointed out by the referee that the great $(k) x$-web $\Sigma$ might have some separating vertices. Then after cutting along separating vertices, we can choose a subgraph of $\Sigma$ which is also a great $(k / 2+\triangle) x$-web containing no separating vertex, because there are at least two subgraphs of $\Sigma$ containing only one separating vertex of $\Sigma$. Then we are able to use the following inequality instead of (1):

$$
(\triangle+2)(v-n)+\triangle n-\left(\frac{k}{2}+\triangle\right) \leq 2 e .
$$

Thus $(\triangle-4) v+6-\triangle \leq k / 2$, which again contradicts that $k \leq \triangle / 2+1$.

Lemma 3.3. $G_{T}$ contains a Scharlemann cycle.

Proof. We separate two cases.

(1) Suppose that there is a vertex $x$ of $G_{Q}$ such that for all labels $y$ at least 2 edges incident to $x$ at $y$ connect $x$ to antiparallel vertices.

Then the conclusion follows from case (1) of the proof of Lemma 5.1 of [Oh].

(2) Here we assume the negation of (1). That is, for each vertex $x$ of $G_{Q}$ there is a label $y(x)$ such that at least $\triangle-1$ edges incident to $x$ at $y(x)$ connect $x$ to parallel vertices.

Let $\Lambda_{Q}$ be an innermost connected component of the subgraph which is obtained from $G_{Q}$ by deleting all edges connecting antiparallel vertices and all separating families of mutually parallel edges. Without loss of generality, we assume that in $\Lambda_{Q}$, every vertex, except possibly one, called a ghost vertex, which is an endpoint of a separating family of mutually parallel edges, has valency at least $(\triangle-2) t+2$. Then $\Lambda_{Q}$ here is very similar to the graph $\Gamma_{Q}$ described in the proof of Theorem 3.2. The same argument as the proof of Theorem 3.2 shows that there is no interior vertex of $\Lambda_{Q}$ of valency at most $\triangle+1$ in $\bar{\Lambda}_{Q}$, and so there is a boundary vertex, $y$, of $\Lambda_{Q}$ which is not a ghost vertex and has valency at most $\triangle-1$ in $\bar{\Lambda}_{Q}$ (here $\bar{\Lambda}_{Q}$ has at most one ghost vertex). By using Lemma 2.2(2) twice, $(\triangle-2) t+2 \leq(\triangle-1)\left(\frac{t}{2}+2\right)$, i.e. $t \leq 4(\triangle-2) /(\triangle-3)$ and $t \neq 6$. Thus $t=8$ when $\triangle=4$. That is, $y$ has valency at least 18 in $\Lambda_{Q}$ and at most 3 in $\bar{\Lambda}_{Q}$. By Lemma 2.2(2) again, these 18 edges consist of 3 families each of which has exactly 6 mutually parallel edges and so contains 2 S-cycles on disjoint label pairs. And adjacent 2 families contain 4 S-cycles on disjoint label pairs, which contradicts Lemma 2.2(1).

Now we are ready to prove Theorem 1.1 when $t \geq 6$.

Proof of Theorem 1.1. Using Proposition 3.1 and Theorem 3.2, we are able to conclude that for all $\{1, \ldots, q\}$-types, $\tau$, there are at least $k t$ faces, i.e. more than $(\triangle / 2) t$ faces, of $G_{T}$ representing $\tau$. Recall that $\widehat{Q}$ is essential in $M(\pi)$ and $G_{T}$ contains a Scharlemann cycle. Now we can apply the same arguments in the context of Sections 4,5 and 6 in [GLu2] where they use the facts that $\triangle=2$ and for every face type $\tau$ there are more than $p$ (the number of boundary components of the other planar surface $P$ ) faces representing $\tau$. In our case we have more than $(\triangle / 2) t$ faces representing $\tau$. In [GLu2] it is shown that there are a vertex $v$ of $G_{Q}$ 
and certain face types such that each face of $G_{T}$ representing such a type contains an edge incident to $v$, and this gives rise to too many edges in $G_{Q}$ incident to $v$. The theorem follows.

\section{Exceptional CaSes $t=2$ OR 4}

This section will be devoted to proving Theorem 1.1 in the special cases $t=2$ and $t=4$. We will make frequent use of the combinatorics of Lemma 2.1 and Lemma 2.3 throughout this section to complete the proof of the main theorem.

Lemma 4.1. If $t=2$ then $\triangle \leq 3$.

Proof. Since $G_{S}$ is a graph with one vertex, say 1, on a Klein bottle, the number of families of mutually parallel edges is at most 3, i.e. this vertex has valency at most 6 in $\bar{G}_{S}$. Hence $\triangle<6$ by Lemma 2.1. Thus there are two cases.

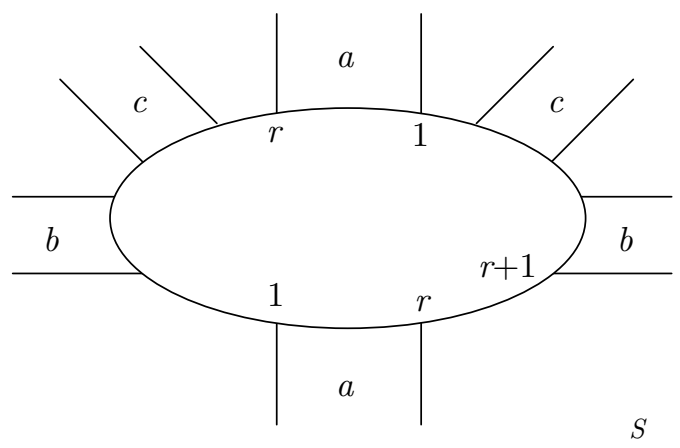

(a)

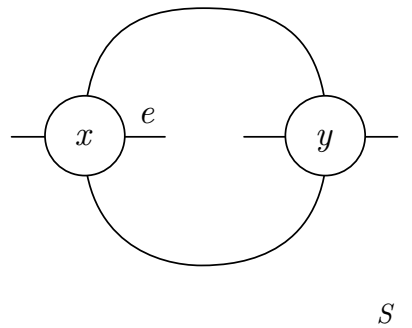

(b)

\section{FiguRe 1}

(1) When $\triangle=4 ; G_{S}$ looks like Figure $1(\mathrm{a})$, where $1<r<q$.

Here only the edges of family $a$ correspond to an orientation-preserving curve on $\widehat{S}$ and hence these edges connect antiparallel vertices of $G_{Q}^{S}$. Each pair of edges labelled $x$ and $r+1-x$ (where $x=1,2, \ldots, r$ ) of family $a$ forms a cycle meeting vertices $x$ and $r+1-x$ in $G_{Q}^{S}$. Choose an innermost such cycle with vertices, say $x$ and $y=r+1-x$, which bounds a disk $D$ such that there is no edge connecting antiparallel vertices in the interior of $D$, as in Figure 1(b). Since in $G_{S}$ these edges are incident to 1 at non-adjacent occurrences of the label $x$, in $G_{Q}^{S}$ they are nonadjacent at vertex $x$. Let $e$ be the edge incident to $x$ that lies in $D$. Let $\Gamma$ be the subgraph of $G_{Q}^{S}$ which consists of all vertices parallel to $x$ in the interior of $D$ and their connecting edges except $e$. Then every vertex of $\Gamma$, except the one which $e$ is incident to, has even valency, namely 4 . This contradicts a property of a graph.

(2) When $\triangle=5$; We see $G_{S}$ as shown in Figure 2(a), where $1<r<\frac{q}{2}$.

Again only the edges of families $a_{1}, a_{2}$ and $a_{3}$ correspond to the edges connecting antiparallel vertices of $G_{Q}^{S}$. As in case (1), each pair of edges of families $a_{1}$ and $a_{3}$ is a cycle meeting two related vertices in $G_{Q}^{S}$. Then there is an innermost cycle with vertices, say $x$ and $y$, which bounds a disk $D$ such that $D$ does not contain the vertices $1, \ldots, r, \frac{q}{2}+1, \ldots, \frac{q}{2}+r$. Now consider the edges incident to $x$ and $y$ in $D$. Since both boundary edges of $D$ are incident to 1 at labels $x$ (and $y$ ) and of the 


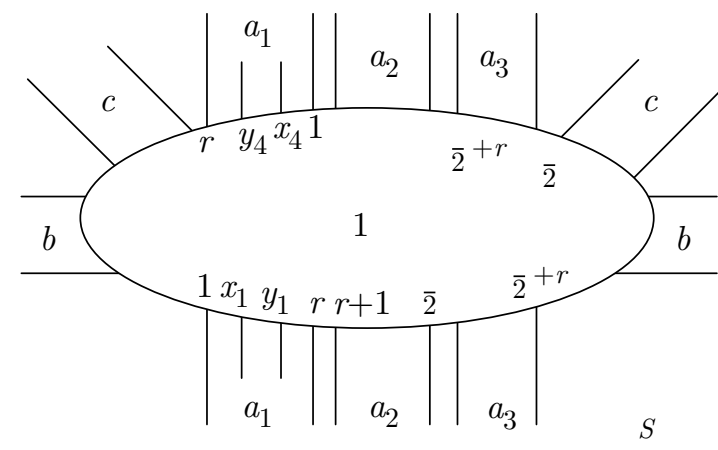

(a)

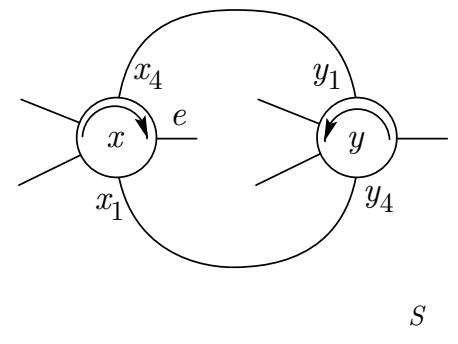

(b)

Figure 2

same family in $G_{S}$, and $x$ and $y$ are antiparallel, if $n$ edges are incident to $x$ in the interior of $D$ then 3-n edges are incident to $y$ in the interior of $D$ where $n=0,1,2$ or 3 (as shown in Figure 2(b) when $n=1$ ). Thus either $x$ or $y$ has odd valency in the interior of $D$. Furthermore for each vertex $v$ in the interior of $D$, only one edge incident to $v$ connects $v$ to an antiparallel vertex (this edge is in family $a_{2}$ in $G_{S}$ ); i.e. exactly the other four edges connect $v$ to parallel vertices. As is done in case (1), we can define a subgraph of $G_{Q}^{S}$, only one of whose vertices has odd valency, a contradiction.

Lemma 4.2. If $t=4$ then $\triangle \leq 3$.

Proof. By an Euler characteristic count, $\bar{G}_{S}$ has at most 6 edges with 2 vertices as shown in Figure 3(a). Hence its double cover $\bar{G}_{T}$ is the graph illustrated in Figure 3 (b) (or the same graph but with the vertices on the middle line labelled,,,+--+ ) because the first and the third vertices come from the same vertex of $\bar{G}_{S}$, i.e. they are antiparallel.

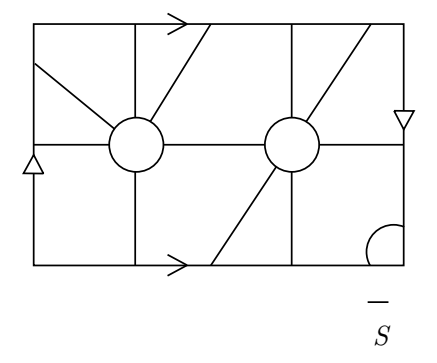

(a)

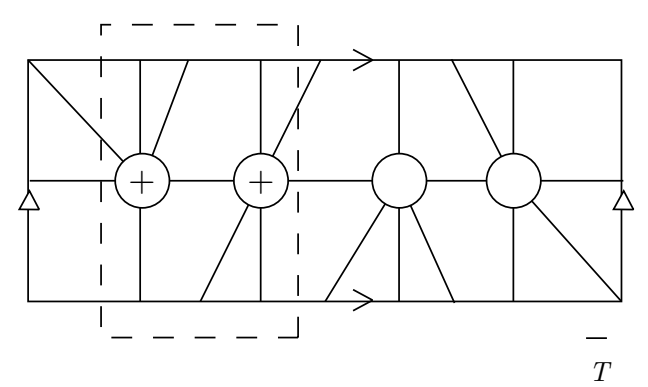

(b)

Figure 3

We may assume that $G_{T}$ contains a part of the graph as shown in Figure 4.

Lemmas 2.1 and 2.3(2) imply the following inequality:

$$
2(q-1)+4\left(\frac{q}{2}+1\right)=4 q+2 \geq \triangle q .
$$




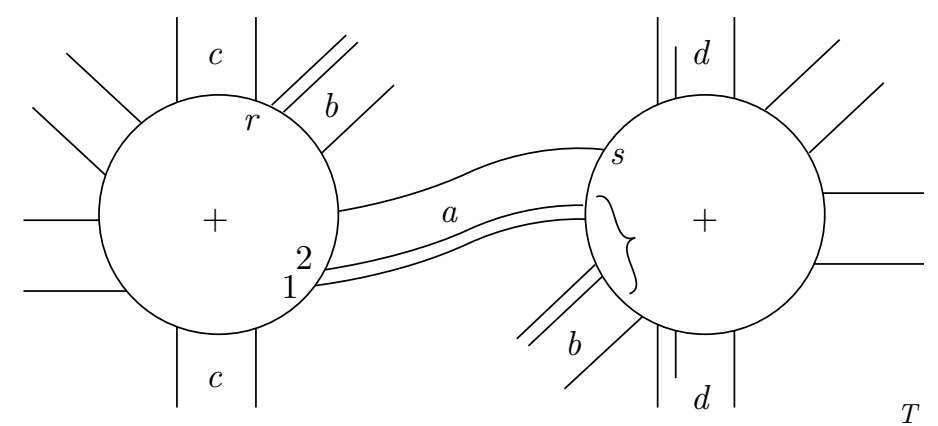

FiguRE 4

Hence $\triangle \leq 4$. Assume that $\triangle=4$. In Figure $4 r$ must be odd by the parity rule.

Suppose that families $a$ and $b$ have a total of $q+2$ edges, i.e. each family has $q / 2+1$ edges. Lemma 2.3(2) implies that each family contains an S-cycle on the side. Thus family $a$ contains $\{1,2\}$ or $\left\{\frac{q}{2}, \frac{q}{2}+1\right\}$ S-cycle and family $b$ contains $\left\{\frac{q}{2}+2, \frac{q}{2}+3\right\}$ or $\{1,2\}$ S-cycle. If both families contain $\{1,2\}$ S-cycles, then we have labels $1,2,1$ and 2 successively on mark $A$ indicated in Figure 4 . It is impossible. Thus $G_{T}$ contains two S-cycles on distinct label pairs in each family, contradicting Lemma $2.3(1)$.

Therefore families $a$ and $b$ have $q$ edges, i.e. $r=1$. Then each family $c$ and $d$ has $q / 2+1$ edges. By Lemma 2.3(1) and (2) again, both families contain $\left\{\frac{q}{2}, \frac{q}{2}+1\right\}$ $\mathrm{S}$-cycles on the side. If 2 edges on the left side of family $d$ form $\left\{\frac{q}{2}, \frac{q}{2}+1\right\}$ S-cycle, then families $a$ and $b$ must have $q-2$ edges. Thus 2 edges on the other side form $\left\{\frac{q}{2}, \frac{q}{2}+1\right\}$ S-cycle, i.e. $s=1$. This implies that the middle 2 edges of family $a$ form an S-cycle which do not have labels $\left\{\frac{q}{2}, \frac{q}{2}+1\right\}$, a contradiction.

\section{REFERENCES}

[BZ1] S. Boyer and X. Zhang, Finite Dehn Surgery On Knots, J. Amer. Math. Soc. 9 (1996), 1005-1050. CMP 96:15

[BZ2] , The Semi-norm and Dehn Filling, preprint.

[BZ3]_ Reducing Dehn Filling and Toroidal Dehn Filling, preprint.

[CGLS] M. Culler, C. Gordon, J. Luecke and P. Shalen, Dehn surgery on knots, Ann. of Math. 125 (1987) 237-300. MR 88a:57026

[Go1] C. Gordon, Dehn surgery on knots, Proc. Int. Congress of Math., Kyoto 1990, 631-642. MR 93e:57006

[Go2] - Boundary slopes of punctured tori in 3-manifolds, preprint.

[GLu1] C. Gordon and J. Luecke, Knots are determined by their complements, J. Amer. Math. Soc. 2 (1989) 371-415. MR 90a:57006a

[GLu2] _ Reducible manifolds and Dehn surgery, Topology 35 (1996), 385-409. CMP 96:10

[GLu3] _ Dehn surgeries on knots creating essential tori, I, Communications in Analysis and Geometry 3 (1995), 597-644. MR 96k:57003

[GLi] C. Gordon and R. Litherland, Incompressible planar surfaces in 3-manifolds, Topology and its Appl. 18 (1984) 121-144. MR 86e:57013

[Ja] W. Jaco, Lectures on three-manifold topology, CBMS Regional Conf. Ser. Math. 43 (1980). MR 81k:57009

[Oh] S. Oh, Reducible and toroidal 3-manifolds obtained by Dehn fillings, Topology and its Appl. 75 (1997), 93-104. CMP 97:05

[Ru] H. Rubinstein, On 3-manifolds that have finite fundamental groups and contain Klein bottles, Trans. Amer. Math. Soc. 251 (1979) 129-137. 
[Th] W. Thurston, Three dimensional manifolds, Kleinian groups, and hyperbolic geometry, Bull. Amer. Math. Soc. 6 (1982) 357-381. MR 83h:57019

[Wu1] Y. Wu, The reducibility of surgered 3-manifolds, Topology and its Appl. 43 (1992) 213218. MR 93e: 57032

[Wu2] _ Dehn fillings producing reducible manifold and toroidal manifold, preprint.

Department of Mathematics, University of Texas at Austin, Austin, Texas 78712

E-mail address: soh@math.utexas.edu

Current address: Department of Mathematics, KAIST, 373-1 Kusungdong Yusunggu, Taejeon, Korea 305-701 